\title{
Exact Penalization of Generalized Nash Equilibrium Problems*
}

\author{
Qin Ba Jong-Shi Pang
}

Original November 18, 2018

\begin{abstract}
This paper presents an exact penalization theory of the generalized Nash equilibrium problem (GNEP) that has its origin from the renowned Arrow-Debreu general economic equilibrium model. While the latter model is the foundation of much of mathematical economics, the GNEP provides a mathematical model of multi-agent non-cooperative competition that has found many contemporary applications in diverse engineering domains. The most salient feature of the GNEP that distinguishes it from a standard non-cooperative (Nash) game is that each player's optimization problem contains constraints that couple all players' decision variables. Extending results for stand-alone optimization problems, the penalization theory aims to convert the GNEP into a game of the standard kind without the coupled constraints, which is known to be more readily amenable to solution methods and analysis. Starting with an illustrative example to motivate the development, the paper focuses on two kinds of coupled constraints, shared (i.e., common) and finitely representable. Constraint residual functions and the associated error bound theory play an important role throughout the development.
\end{abstract}

\section{Introduction}

The Generalized Nash Equilibrium Problem (GNEP) extends the classical Nash Equilibrium Problem (NEP) by allowing individual players' constraints, in addition to the payoff functions, to depend on rival players' strategies. This extension adds considerably descriptive and explanatory power for the GNEP to model non-cooperative competition among multiple selfish decision-making agents. Since its introduction in the 1950's [13, 1] (where the terms social equilibrium and abstract economy were used), the GNEP has risen beyond its mathematical-economic origin and become a common paradigm for various engineering disciplines. This model fits well in a non-cooperative game setting where all players share some common resources or limitations. Thus some of the players' constraints are coupled; these are in addition to the players' private constraints that do not contain the rival players' decision variables. Sources of the GNEP include infrastructure systems such as communication or radio systems [49, 50], power grids [57, 35], transportation networks [5], modern traffic systems with e-hailing services [4], supply and demand constraints for transportation systems [54], and pollution quota for environmental application [40, 7]. We refer the readers to [22, 27, 30] for more detailed surveys on this active research topic and its many applications. The four chapters by Facchinei published in [12, pages 151-188] contain a historical account of the GNEP, provide many references, and summarize the advances up to the year 2012.

\footnotetext{
${ }^{*}$ Both authors are affiliated with the Daniel J. Epstein Department of Industrial and Systems Engineering, University of Southern California, Los Angeles, California 90089-0193, U.S.A. Emails: qba@usc.edu, jongship@usc.edu . This work was based on research supported by the National Science Foundation under grant IIS-1632971 and by the Air Force Office of Scientific Research under Grant Number FA9550-18-1-0382.
} 
Due to the presence of the coupling constraints, solving a GNEP is a significantly more challenging task than solving a NEP. Depending on the types of the coupling constraints, a GNEP can be divided into two categories. The first category is the shared-constrained case, where the coupling constraints are common to all players. [In the literature, this case has also been called jointly constrained, or common constrained; we adopt the "shared-constrained" terminology in this paper.] Many engineering problems cited in last paragraph are of this type. The second type is more general in which the coupling constraints can be specific to each player and different among the players. Due to the relative simplicity and direct connections to many engineering problems, shared-constrained GNEPs receive most of the attention in the literature. Particularly, much effort has been spent on obtaining a special kind of equilibrium solutions, called variational equilibrium, under various settings and assumptions. Such an equilibrium has its origin from [52]; the term "variational equilibrium" was coined in [22] and further explored in many papers that include [21, 27, 22, 42] where additional references can be found. In turn, the adjective "variational" was employed to express that this kind of equilibria of a GNEP can be described as solutions of a variational inequality [34, 25] obtained by concatenating the first-order conditions of the players' optimization problems. Alternatively, a Nikaido-Isoda-type bi-function [46] has been used extensively [41, 39, 56] as an attempt to formulate a GNEP as a single optimization problem. In general, the existence of a variational equilibrium often relies on the convexity and certain boundedness assumptions and suitable constraint qualifications [27]. While computationally simpler and providing a special kind of solutions, a variational equilibrium does not always have a clear advantage over a non-variational equilibrium solution. It is hence difficult to argue that special focus should be placed on finding such a particular equilibrium. Nevertheless, computing a non-variational equilibrium solution is more complex and less understood. Yet there has been some literature on this subject. For example, an extension of the class of variational equilibria is studied in [31, 44], and algorithms aiming at obtaining the entire set of solutions of special class of GNEPs are considered in [28, 19, 3. The paper [53] discusses the numerical solution of an affine GNEP by Lemke's method with an attempt to compute generalizations of variational equilibria.

With the goal of exploiting the advances in the NEPs, this paper revisits and sharpens an exact penalization theory for GNEPs whereby the coupled constraints, shared or otherwise, are moved from the constraints to the objective functions via constraint residual functions scaled by a penalty parameter. Dating back to the beginning of nonlinear programming [29], exact penalization for optimization has been a subject of intensive study in the early days of optimization (see [33]) and much research has been undertaken by the Italian School led by Di Pillo, Facchinei and Grippo [14, 15, 16, 17, 18. Exemplified by these works and references therein, this theory deals largely with problems whose constraints are defined by differentiable inequalities and relies on the multipliers of these constraints. The papers [9, 8] discuss how penalty functions can be used to design numerical methods for solving nonlinear programs that have been implemented in the very successful KNITRO software [10]. For the GNEP, exact penalty results are derived in [26, 24] which also discuss algorithmic design. The paper [23] discusses extensive computational rules for updating the penalty parameter and reports numerical results with the resulting algorithms. Contrary to exact penalization, inexact, or asymptotic penalization also penalizes the constraints but the penalty parameter is required to tend to infinity to recover a solution of the given problem. A fairly simple iterative inexact-penalty based method for solving the GNEP was discussed in [48]; this method cast the game as a quasi variational inequality obtained by concatenating the first-order optimality conditions of the players' nonlinear programs. The recent papers [36, 37] expanded the latter paper and discussed augmented Lagrangian and exact penalty methods for the GNEP and the more general quasi-variational inequality, respectively. 
In contrast to the multiplier-based exact penalization, the principle of exact penalization, first established in [11, Proposition 2.4.3] and subsequently generalized in [25, Theorem 6.8.1] deals with the exact penalization of an abstract constraint set. The latter result makes it clear that this theory is closely connected to the theory of error bounds [6, 43] and [25, Chapter 6]. To date, this approach has not been applied to the GNEP; part of the goal of this paper is to establish how exact penalty results for the GNEP can be derived based on an error-bound theory. Unlike a standalone optimization problem, a GNEP has multiple inter-connected optimization problems each with an objective function of its own; thus while exact penalty results for the players' optimization problems are available, simply putting them together does not readily yield an exact penalty result for a GNEP. The inter-connection between the players' optimization problems will need to be accounted for when the coupling constraints are penalized. Furthermore, while most literature on the exact penalization of the GNEP considers only coupling inequality constraints, see e.g., [23, 24, 31, 36]; another goal of this paper is to bring inequality coupling constraints to the study of exact penalization. By means of an illustrative example, we show that care is needed in choosing the constraint residual functions in order for the conversion to a penalized NEP to be valid. This example also paves the way for the theory to be developed subsequently. It is worth noting that the $\ell_{q}$ residual functions for $q \in(1, \infty)$ present certain advantages in guaranteeing exact penalization of GNEP. This class of residual functions is used in [24] and [12, Chapter 3 Section 4.1]. This paper also takes advantage of them in finitely representable cases; Sections 4 and 5 therein elucidate the advantageous functional properties of $\ell_{q}$ residual functions in exact penalization.

\section{Preliminary Discussion and Review}

In this section, we present the setting of this paper and fix the notations to be used throughout. We begin with the definition of the GNEP, then review some relevant concepts and the principle of exact penalization for an optimization problem, and finally end with an illustration of this principle applied to a simple GNEP and a preliminary result.

\subsection{The GNEP}

Formally, a GNEP consists of $N$ selfish players each of whom decides a strategy with the goal to minimize (or maximize) his/her cost (or payoff). Let $x^{\nu} \in \mathbb{R}^{n_{\nu}}$ denote the decision of player $\nu \in[N] \triangleq\{1, \cdots, N\}$. Anticipating the rival players' strategies $x^{-\nu} \triangleq\left(x^{\nu^{\prime}}\right)_{\nu^{\prime} \neq \nu}$, player $\nu \in[N]$ solves the following minimization problem in his/her own variable $x^{\nu}$ :

$$
\begin{array}{ll}
\underset{x^{\nu} \in C^{\nu}}{\operatorname{minimize}} & \theta_{\nu}\left(x^{\nu}, x^{-\nu}\right) \\
\text { subjectto } & x^{\nu} \in X^{\nu}\left(x^{-\nu}\right),
\end{array}
$$

where $\theta_{\nu}\left(x^{\nu}, x^{-\nu}\right)$ denotes the cost function of player $\nu$ as a function of the pair $\left(x^{\nu}, x^{-\nu}\right)$; the constraints are of two types: those described by the private strategy set $C^{\nu} \subseteq \mathbb{R}^{n_{\nu}}$ and the others described by the coupled constraint set $X^{\nu}\left(x^{-\nu}\right) \subseteq \mathbb{R}^{n_{\nu}}$ that depends on the rival players' strategy tuple $x^{-\nu}$. [Writing $\left(x^{\nu}, x^{-\nu}\right)$ in the argument of $\theta_{\nu}(\bullet)$ is to emphasize player $\nu$ 's strategy $x^{\nu}$ and does not mean that the tuple $x \triangleq\left(x^{\nu}\right)_{\nu=1}^{N} \in \mathbb{R}^{n}$, where $n \triangleq \sum_{\nu \in[N]} n_{\nu}$, is reordered such that $x^{\nu}$ is the first block.] This setup clearly indicates that both the objective function and constraint set of each player's optimization problem are coupled by all players' strategies. Let $\theta(x) \triangleq\left(\theta_{\nu}\left(x^{\nu}, x^{-\nu}\right)\right)_{\nu=1}^{N}$

be the concatenation of the players' objective functions; $C \triangleq \prod_{\nu \in[N]} C^{\nu}$ be the concatenation of the 
players' private constraints, and $X: \mathbb{R}^{n} \rightarrow \mathbb{R}^{n}$ with $X(x) \triangleq \prod_{\nu \in[N]} X^{\nu}\left(x^{-\nu}\right)$ be the multifunction concatenating the players' coupled constraints. We use the triplet $(C, X ; \theta)$ to denote the GNEP with players' optimization problems defined by (1). A tuple $x^{*} \triangleq\left(x^{*, \nu}\right)_{\nu \in[N]}$ is a Nash Equilibrium (NE) of this game if $x^{*, \nu} \in \underset{x^{\nu} \in C^{\nu} \cap X^{\nu}\left(x^{*,-\nu}\right)}{\operatorname{argmin}} \theta\left(x^{\nu}, x^{*,-\nu}\right)$ for all $\nu \in[N]$.

By considering $X^{\nu}$ as a multifunction from $\mathbb{R}^{n_{-\nu}}$ into $\mathbb{R}^{n_{\nu}}$, where $n_{-\nu} \triangleq n-n_{\nu}$, the constraint $x^{\nu} \in X^{\nu}\left(x^{-\nu}\right)$ is equivalent to the membership that $\left(x^{\nu}, x^{-\nu}\right)$ belongs to the graph of $X^{\nu}$, which is denoted by $\operatorname{graph}\left(X^{\nu}\right) \subseteq \mathbb{R}^{n}$. An important special case of the $\operatorname{GNEP}(C, X ; \theta)$ is when these graphs are all equal to a common set $D \subseteq \mathbb{R}^{n}$. This is the case of common coupled constraints, which we call shared constraints. We denote this special case by the triplet $(C, D ; \theta)$. If each function $\theta_{\nu}\left(\bullet, x^{-\nu}\right)$ is differentiable and convex and the set $C \cap D$ is convex, then a special kind of NE of the GNEP $(C, D ; \theta)$ is that derived from the variational inequality, denoted by VI $(C \cap D ; \Theta)$, of finding a vector $\bar{x} \in C \cap D$ such that $(y-\bar{x})^{\mathrm{T}} \Theta(\bar{x}) \geq 0$ for all $y \in C \cap D$, where $\Theta(x) \triangleq\left(\nabla_{x^{\nu}} \theta_{\nu}(x)\right)_{\nu \in[N]}$. Such a special NE is a called a variational equilibrium. When the graphs $\operatorname{graph}\left(X^{\nu}\right)$ are not the same, NEs of the GNEP $(C, X ; \theta)$ are related to solutions of a quasi-variational inequality; see e.g. 48, for details.

Another important special case of the $\operatorname{GNEP}(C, X ; \theta)$ is when each set $X^{\nu}\left(x^{-\nu}\right)$ is finitely representable, i.e., when

$$
X^{\nu}\left(x^{-\nu}\right) \triangleq\left\{x^{\nu} \in \mathbb{R}^{n_{\nu}} \mid g^{\nu}\left(x^{\nu}, x^{-\nu}\right) \leq 0 \text { and } h^{\nu}\left(x^{\nu}, x^{-\nu}\right)=0\right\}
$$

for some functions $\left(g^{\nu}, h^{\nu}\right): \mathbb{R}^{n} \rightarrow \mathbb{R}^{m_{\nu}+p_{\nu}}$ with some positive integers $m_{\nu}$ and $p_{\nu}$. Typically, such constraint functions $g^{\nu}$ and $h^{\nu}$ are such that $g^{\nu}\left(\bullet, x^{-\nu}\right)$ is differentiable and each of its component functions $g_{i}^{\nu}\left(\bullet, x^{-\nu}\right)$ is convex and $h^{\nu}\left(\bullet, x^{\nu}\right)$ is affine; in this case, the set $X^{\nu}\left(x^{-\nu}\right)$ is both closed and convex; see Section 5 .

A road map. For the GNEP $(C, X ; \theta)$ and its special cases, exact penalization aims to convert these games with coupled constraints to standard games whereby the constraint set $X^{\nu}\left(x^{-\nu}\right)$ for each player is moved to the objective function via a residual function (see definition in the next subsection) scaled by a penalty parameter $\rho$, thereby obtaining a NEP $\left(C ; \theta_{\rho}\right)$ for a concatenated penalized objective $\theta_{\rho}$, whose solution is a solution of the given GNEP. Notice that we retain the private constraints described by the set $C^{\nu}$ in each player's optimization problem. Thus this is a type of partial exact penalization [24]. The development in the rest of this paper is as follows. We first present an illustrative example which reveals important insights into exact penalization of GNEP, then consider the penalization of the GNEP $(C, D ; \theta)$ with common coupled constraints and general closed convex sets $C$ and $D$ with no particular structure. The latter part is concluded with the case where $D$ is finitely representable by convex inequalities and linear equalities. Finally, we discuss the GNEP $(C, X ; \theta)$ by penalizing the coupled constraints defined as in (2). We should note that the literature on exact penalization of the GNEP has dealt largely with the latter problem under constraint qualifications, such as the sequentially bounded CQ [26, 48] and the Extended Mangasarian-Fromovitz constraint qualification (EMFCQ) [23, 24, 31, 36]. In this paper, we have provided results for exact penalization of GNEP under a strong descent assumption and a Lipschitz error bound assumption for residual functions, see Proposition 3 . Theorem 5 and Corollary 8, and a Slater-type condition for the coupled constraints, see Theorem 10 and 11 . 


\subsection{Relevant concepts}

We introduce in this subsection some relevant concepts that are used later. A function $\Phi: \mathbb{R}^{n} \rightarrow \mathbb{R}^{m}$ is said to be Lipschitz continuous on a set $S \subseteq \mathbb{R}^{n}$ if, for some positive scalar Lip $_{\Phi}$, the following holds:

$$
|\Phi(x)-\Phi(y)| \leq \operatorname{Lip}_{\Phi}\|x-y\|_{2}, \quad \forall x, y \in S .
$$

This is also referred to as Lipschitz continuity with constant $\operatorname{Lip}_{\Phi}$. In addition, $\Phi$ is said to be Lipschitz continuous near a point $x$ if, for some $\varepsilon>0, \Phi$ is Lipschitz continuous in the $\varepsilon$ neighborhood of $x$. We hence say that $\Phi$ is locally Lipschitz continuous on $S$ if it is Lipschitz continuous near every point in $S$. The directional derivative of $\Phi$ at a point $x \in \mathbb{R}^{n}$ in a direction $d \in \mathbb{R}^{n}$ is defined to be

$$
\Phi^{\prime}(x ; d) \triangleq \lim _{\tau \downarrow 0} \frac{\Phi(x+\tau d)-\Phi(x)}{\tau}
$$

if the limit exists. If the above limit exists for all $d \in \mathbb{R}^{n}, \Phi$ is said to be directionally differentiable at $x$. It is clear that if $\Phi$ is Lipschitz continuous with constant $\operatorname{Lip}_{\Phi}$ near $\bar{x}$, then $\left\|\Phi^{\prime}(\bar{x} ; d)\right\|$ (if the derivative exists) is bounded by $\operatorname{Lip}_{\Phi}\|d\|$ for all $d$.

For two given closed subsets $S$ and $T$ of $\mathbb{R}^{n}$, a function $r_{S}: \mathbb{R}^{n} \rightarrow \mathbb{R}_{+}$is a $T$-residual function of $S$ if for all $x \in T$, it holds that $r_{S}(x)=0$ if and only if $x \in S$, or equivalently if and only if $x \in S \cap T$. Note that this definition allows for $S$ and $T$ to have an empty intersection; in this case, the residual function $r_{S}$ is positive on $T$. Such a residual function $r_{S}(x)$ is said to yield a $T$-Lipschitz error bound of $S$ if there exist a constant $\gamma>0$ such that

$$
\operatorname{dist}(x ; S \cap T) \leq \gamma r_{S}(x), \quad \forall x \in T,
$$

where $\operatorname{dist}(x ; Z) \triangleq \underset{y \in Z}{\operatorname{minimum}}\|y-x\|_{2}$ is the distance function to a closed set $Z$ under the Euclidean norm. These definitions extend the usual concepts of a residual function and associated error bound of a set $S$ which pertain to the case when $T=\mathbb{R}^{n}$. In essence, we are interested in such quantities only for elements in the subset $T$; thus the restriction to the latter set. If $S$ is finitely representable defined by the $m$-dimensional vector function $g$ and the $p$-dimensional vector function $h$ as in (2), we define the $\ell_{q}$ residual function of $S$ for a given $q \in[1, \infty]$ as

$$
r_{q}(x) \triangleq\left\|\left(\begin{array}{c}
g^{+}(x) \\
h(x)
\end{array}\right)\right\|_{q}
$$

where $g^{+}(x) \triangleq \max (g(x), 0)$ and $\|\bullet\|_{q}$ is the $\ell_{q}$-norm of vectors. If each $g_{i}, i \in[m]$ is convex and $h$ is affine, then $r_{q}$ is convex for all $1 \leq q \leq \infty$. Moreover, if these defining functions are differentiable, then for $q \in(1, \infty), r_{q}(x)$ is differentiable at $x \notin S$. Its gradient is given as follows:

$$
\nabla r_{q}(x)=\frac{1}{r_{q}^{q-1}(x)}\left[\sum_{i \in[m]}\left(g_{i}^{+}(x)\right)^{q-1} \nabla g_{i}(x)+\sum_{j \in[p]}\left|h_{j}(x)\right|^{q-1}\left(\operatorname{sgn} h_{j}(x)\right) \nabla h_{j}(x)\right],
$$

where $\operatorname{sgn}(\bullet)$ is the sign function of a scalar defined to be 1 for positive numbers, -1 for negative numbers, and 0 for 0 . In the same case, the function $r_{q}(x)$ is directional differentiable at all $x \in S$ and its directional derivative is given by:

$$
r_{q}^{\prime}(x ; d)=\left(\sum_{i: g_{i}(x)=0}\left[\max \left(\nabla g_{i}(x)^{\mathrm{T}} d, 0\right)\right]^{q}+\sum_{j: h_{j}(x)=0}\left|\nabla h_{j}(x)^{\mathrm{T}} d\right|^{q}\right)^{1 / q},
$$


As for $q=1, r_{1}(x)$ is not differentiable but directional differentiable and its directional derivative is given by:

$$
\begin{aligned}
r_{1}^{\prime}(x ; d)= & \sum_{i: g_{i}(x)>0} \nabla g_{i}(x)^{\mathrm{T}} d+\sum_{i: g_{i}(x)=0} \max \left(\nabla g_{i}(x)^{\mathrm{T}} d, 0\right)+ \\
& \sum_{j: h_{j}(x)=0}\left|\nabla h_{j}(x)^{\mathrm{T}} d\right|+\sum_{j: h_{j}(x) \neq 0}\left(\operatorname{sgn} h_{j}(x)\right) \nabla h_{j}(x)^{\mathrm{T}} d .
\end{aligned}
$$

\subsection{Partial exact penalization}

Exact penalization of GNEPs has its origin from its counterpart for solving constrained optimization problems. As such, we begin by considering the following general constrained optimization problem:

$$
\underset{x \in W \cap S}{\operatorname{minimize}} f(x),
$$

where $W$ and $S$ represent two sets of constraints with $S$ considered more complex than $W$. By penalizing the violation of membership in $S$, a penalty formulation transforms $(8)$ into the following penalized optimization problem:

$$
\underset{x \in W}{\operatorname{minimize}} f(x)+\rho r_{S}(x)
$$

where $\rho>0$ is a penalty parameter to be specified and $r_{S}(x)$ is a $W$-residual function of the set $S$. The penalization is said to be exact for (8) if there exists a positive scalar $\bar{\rho}$ such that, for all $\rho \geq \bar{\rho}$, every global minimizer of the penalized problem is also a global minimizer of (8) and vice versa. Note: Dealing with minimizers, this definition is a classical one. In this regard, the definition is computationally most meaningful when both (8) and $(9)$ are convex programs. Otherwise, the equivalence between the minimizers is a conceptual property that offers guidance to solve constrained problems in practical algorithms via relaxations of some constraints. Subsequently, when we apply this optimization theory to the GNEP, we will make some needed convexity assumptions so that we can freely talk about minimizers.

It is important to note that the penalized problem (9) is different from the usual one as discussed in [25, Section 6.8] which is:

$$
\underset{x \in W}{\operatorname{minimize}} f(x)+\rho r_{S \cap W}(x),
$$

where $r_{S \cap W}(x)$ is a residual function of the intersection $S \cap W$ in the unrestricted sense. As such, while the result below is similar to [25, Theorem 6.8.1], they differ in several ways: one, the penalized problems are different; two, the error bound conditions are different; and three, the result below does not pre-assume the solvability of either (8) or (9). This is in contrast to the solvability assumption that is needed for one inclusion of the two argmin sets in the cited theorem. For completeness, we offer a detailed proof of the result below which does not explicitly assume convexity of the functions involved.

Theorem 1. Consider the nonlinear program (8) with closed sets $W$ and $S$ and a Lipschitz continuous objective function $f$ with constant $\operatorname{Lip}_{f}>0$ on the set $W$. Assume that $S \cap W \neq \emptyset$. Let $r_{S}(x)$ be a $W$-residual function of the set $S$ satisfying a $W$-Lipschitz error bound for the set $S$ with constant $\gamma>0$; i.e., $r_{S}(x) \geq \gamma \operatorname{dist}(x ; S \cap W)$ for all $x \in W$. Then for all $\rho>\operatorname{Lip}_{f} / \gamma$,

$$
\underset{x \in S \cap W}{\operatorname{argmin}} f(x)=\underset{x \in W}{\operatorname{argmin}} f(x)+\rho r_{S}(x) .
$$


Proof. In the following two-part proof, we may assume without loss of generality that both argmin sets are nonempty. We first show that the left-hand argmin is a subset of the right-hand argmin. Let $\bar{x}$ be an element of $\operatorname{argmin} f(x)$. Let $x \in W$ be arbitrary. Let $\widehat{x}$ be a vector in $S \cap W$ such that $\|x-\widehat{x}\|_{2}=\operatorname{dist}(x ; S \cap W)$. We have

$$
\begin{aligned}
f(x)+\rho r_{S}(x) & \geq f(x)+\rho \gamma\|x-\widehat{x}\|_{2} & & \text { by the } W \text {-Lipschitz error bound } \\
& \geq f(\widehat{x})-\operatorname{Lip}_{f}\|x-\widehat{x}\|_{2}+\rho \gamma\|x-\widehat{x}\|_{2} & & \text { by the Lipschitz continuity of } f \\
& \geq f(\widehat{x}) & & \text { by the choice of } \rho \\
& \geq f(\bar{x})=f(\bar{x})+\rho r_{S}(\bar{x}) & & \text { by the optimality of } \bar{x} .
\end{aligned}
$$

This establishes one inclusion. For the converse, let $x^{*} \in \operatorname{argmin} f(x)+\rho r_{S}(x)$. It suffices to show that $x^{*} \in S$. Let $\widetilde{x}$ be a vector in $S \cap W$ such that $\left\|x^{*}-\widetilde{x}\right\|_{2}=\operatorname{dist}\left(x^{*} ; S \cap W\right)$. We have

$$
\begin{aligned}
f(\widetilde{x}) & \geq f\left(x^{*}\right)+\rho r_{S}\left(x^{*}\right) & & \text { by the optimality of } x^{*} \\
& \geq f\left(x^{*}\right)+\rho \gamma\left\|x^{*}-\widetilde{x}\right\|_{2} & & \text { by the } W \text {-Lipschitz error bound } \\
& \geq f(\widetilde{x})-\operatorname{Lip}_{f}\left\|x^{*}-\widetilde{x}\right\|_{2}+\rho \gamma\left\|x^{*}-\widetilde{x}\right\|_{2} & & \text { by the Lipschitz continuity of } f \\
& \geq f(\widetilde{x}) & & \text { by the choice of } \rho .
\end{aligned}
$$

Therefore, $x^{*}=\widetilde{x}$, establishing the claim and the equality of the two sets of minimizers.

We will apply Theorem 1 to the $\operatorname{GNEP}(C, X ; \theta)$ by penalizing the coupled constraint set $X^{\nu}\left(x^{-\nu}\right)$ in each player $\nu^{\prime}$ 's optimization problem. Letting $C^{-\nu} \triangleq \prod_{\nu^{\prime} \neq \nu} C^{\nu^{\prime}}$ and $r_{\nu}\left(\bullet, x^{-\nu}\right)$ be a $C^{\nu}$-residual function of the set $X^{\nu}\left(x^{-\nu}\right)$ for given $x^{-\nu} \in C^{-\nu}$, we obtain the following penalized subproblem for player $\nu$ with respect to given $x^{-\nu}$ :

$$
\underset{x^{\nu} \in C^{\nu}}{\operatorname{minimize}} \theta_{\nu}\left(x^{\nu}, x^{-\nu}\right)+\rho r_{\nu}\left(x^{\nu}, x^{-\nu}\right)
$$

where $\rho>0$ is a penalty parameter which we take for simplicity to be the same for all players. Let $r_{X}(x) \triangleq\left(r_{\nu}(x)\right)_{\nu=1}^{N}$ and $\theta_{\rho ; X} \triangleq \theta+\rho r_{X}$. Then the optimization problems (11) concatenated for all $\nu \in[N]$ define the game $\left(C ; \theta_{\rho ; X}\right)$ which is clearly a NEP. We say that exact penalization holds for the $\operatorname{GNEP}(C, X ; \theta)$ with residual function $r_{X}$ if there exists a scalar $\bar{\rho}>0$ such that, for all $\rho \geq \bar{\rho}$, every equilibrium solution of the $\operatorname{NEP}\left(C ; \theta_{\rho ; X}\right)$ is also an equilibrium solution of the GNEP $(C, X ; \theta)$ and vise versa. The following example drawn from [27] illustrates this penalization.

Example 2. Consider the shared-constrained $\operatorname{GNEP}(C, D ; \theta)$ with the following specifications: $N=2 ; n_{1}=n_{2}=1 ; \theta_{1}(x)=\left(x_{1}-1\right)^{2} ; \theta_{2}(x)=\left(x_{2}-1 / 2\right)^{2} ; C=\mathbb{R}^{2}$, and $D=\left\{x \in \mathbb{R}^{2} \mid x_{1}+x_{2} \leq 0\right\}$. The set of equilibria of this GNEP is $\{(\alpha, 1-\alpha) \mid 1 / 2 \leq \alpha \leq 1\}$. The set of variational equilibria of the GNEP is a singleton containing $(3 / 4,1 / 4)$. With $\rho>0$ as the penalty parameter, the penalized $\mathrm{NEP}$ is defined by the following two univariate optimization problems:

$$
\underset{x_{1}}{\operatorname{minimize}}\left(x_{1}-1\right)^{2}+\rho\left(x_{1}+x_{2}-1\right)^{+} \underset{x_{2}}{\operatorname{minimize}}\left(x_{2}-\frac{1}{2}\right)^{2}+\rho\left(x_{1}+x_{2}-1\right)^{+}
$$

that employ the common residual function $r_{D}(x)=\left(x_{1}+x_{2}-1\right)^{+}$. The set of equilibria of the latter NEP is obtained by investigating the three cases shown in Table 1. From the table, we conclude that the given GNEP has an exact penalization as a standard NEP. 


\begin{tabular}{cccc}
\hline & $x_{1}^{*}$ & $x_{2}^{*}$ & equilibria set for $\rho \geq 1$ \\
\hline$x_{1}+x_{2}>1$ & $1-\rho / 2$ & $3 / 2-\rho$ & $\emptyset$ \\
$x_{1}+x_{2}<1$ & 1 & $3 / 2$ & $\emptyset$ \\
$x_{1}+x_{2}=1$ & {$[1-\rho / 2,1]$} & {$[1 / 2-\rho / 2,1 / 2]$} & $\{(\alpha, 1-\alpha) \mid 1 / 2 \leq \alpha \leq 1\}$ \\
\hline
\end{tabular}

Table 1: Three cases of the penalized problem

The primary question this paper aims at addressing is under what conditions exact penalization holds for the GNEP $(C, X ; \theta)$ and its special cases. While various exact penalization results exist for the optimization problem (8) in the literature, stand-alone results on the GNEP are not as many; they are mostly embedded in methods for solving the game under some CQs without necessarily claiming the exactness of the penalization. A main difficulty in extending the penalty results for an optimization problem to the GNEP lies in the fact that while each player's optimization problem (1) may have an exact penalty equivalent, the penalty parameter for player $\nu$ 's problem in principle depends on the rivals' strategy tuple $x^{-\nu}$; this makes it difficult for a uniform penalty parameter to exist for all players in an NEP formulation. A related point is highlighted by the proof of the converse inclusion in Theorem 1 which suggests that the feasibility of a solution of the penalized NEP is key for that solution to be a solution of the original un-penalized game. This point was made clear in [26, Theorem 1] and the discussion that follows it where an explanation was offered. We formally state this requirement in the first part of the following preliminary result whose proof we omit.

Proposition 3. Suppose that $C$ and the graph of the constraint multifunction $X$ are closed sets. Let $r_{\nu}\left(\bullet, x^{-\nu}\right)$ be a $C^{\nu}$-residual function of the set $X^{\nu}\left(x^{-\nu}\right)$, for all $x^{-\nu} \in C^{-\nu}$. The following two statements hold:

(a) Let $x^{*}$ be a solution of the penalized NEP $\left(C ; \theta_{\rho ; X}\right)$ for some $\rho>0$. Then $x^{*}$ is a solution of the GNEP $(C, X ; \theta)$ if and only if $x^{*}$ belongs to the graph of the multifunction $X$; i.e., $x^{*, \nu} \in X^{\nu}\left(x^{*,-\nu}\right)$ for all $\nu \in[N]$.

(b) Suppose that for every $x^{-\nu} \in C^{-\nu}, C^{\nu} \cap X^{\nu}\left(x^{-\nu}\right) \neq \emptyset, \theta_{\nu}\left(\bullet, x^{-\nu}\right)$ is Lipschitz continuous with constant $\operatorname{Lip}_{\nu}$ on the set $C^{\nu}$, and $r_{\nu}\left(\bullet, x^{-\nu}\right)$ provides a $C^{\nu}$-Lipschitz error bound with constant $\gamma_{\nu}>0$ for the set $X^{\nu}\left(x^{-\nu}\right)$. Then for all $\rho>\max _{\nu \in[N]} \operatorname{Lip}_{\nu} / \gamma_{\nu}$, every equilibrium solution of the penalized NEP $\left(C ; \theta_{\rho ; X}\right)$ is an equilibrium solution of the $\operatorname{GNEP}(C, X ; \theta)$ and vice versa.

One of the main weakness of part (b) of the above proposition is the assumption that $C^{\nu} \cap X^{\nu}\left(x^{-\nu}\right)$ is nonempty for all $x^{-\nu}$ in $C^{-\nu}$. The invalidity of this assumption contributes to inequality between the set of penalized NE and that of the NE of the GNEP; see parts (a) of Example 4 below. Subsequently, we will provide alternative conditions that bypass this assumption; see Theorem 5 and 11 .

\section{An Illustrative Example}

Before presenting the main results, we offer an example that summarizes several important features in the penalization of the shared-constrained $\operatorname{GNEP}(C, D ; \theta)$ by residual functions of the common constraint set $D$. This example provides the basis for the subsequent results that are motivated by the various penalized NEPs discussed therein. 
Example 4. Consider the share-constrained $\operatorname{GNEP}(C, D ; \theta)$ with the following specifications: $N=2 ; n_{1}=n_{2}=1 ; \theta_{1}(x)=-x_{1} ; \theta_{2}(x)=-x_{2} ; C=[0,4] \times[0,4] ; D=\left\{x \in \mathbb{R}^{2} \mid g_{i}(x) \leq 0, i=\right.$ $1,2,3\}$; where $g_{1}(x)=x_{1}+x_{2}-2, g_{2}(x)=2 x_{1}-x_{2}-2$, and $g_{3}=-x_{1}+2 x_{2}-2$. The constraint set $C \cap D$ is shown as the gray area in Figure 1. It is easy to see that every point on the line segment between $(4 / 3,2 / 3)$ and $(2 / 3,4 / 3)$ (i.e., the solid dark line segment in the figure) is a NE of this GNEP, for which we illustrate various penalizations as follows.

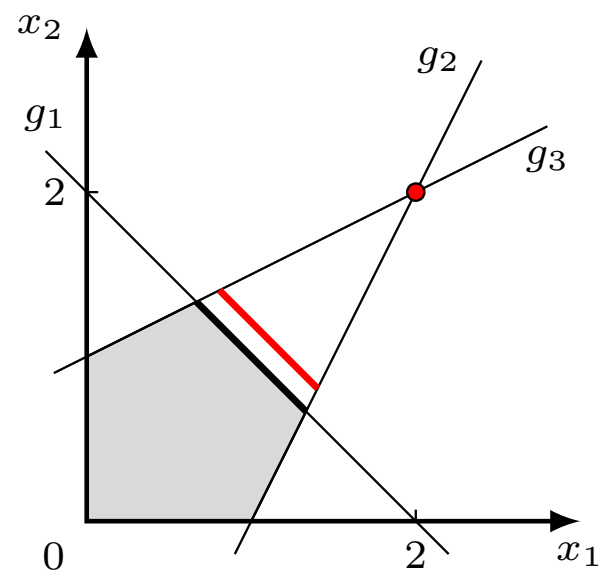

Figure 1: Graph illustration for Example 4

(a) $\ell_{1}$ penalization is not exact. Using the $\ell_{1}$ residual function $r_{1}(x)=\left\|g^{+}(x)\right\|_{1}$, the penalized version of the $\operatorname{GNEP}(C, D ; \theta)$ is the $\operatorname{NEP}\left(C ; \theta+\rho r_{1}\right)$. While the $\ell_{1}$ residual function is a widely used residual function that is proven to be effective for exact penalization of many nonlinear programming problems, this example shows that it does not work generally for exact penalization of even shared, linearly constrained GNEPs. Specifically, we can find an equilibrium solution of the penalized NEP $\left(C ; \theta+\rho r_{1}\right)$ but not one of the $\operatorname{GNEP}(C, D ; \theta)$. The two players' optimization problems for the latter NEP are as follows:

$$
\begin{aligned}
& \underset{0 \leq x_{1} \leq 4}{\operatorname{minimize}}-x_{1}+\rho\left(\left[x_{1}+x_{2}-2\right]^{+}+\left[2 x_{1}-x_{2}-2\right]^{+}+\left[-x_{1}+2 x_{2}-2\right]^{+}\right) \\
& \underset{0 \leq x_{2} \leq 4}{\operatorname{minimize}}-x_{2}+\rho\left(\left[x_{1}+x_{2}-2\right]^{+}+\left[2 x_{1}-x_{2}-2\right]^{+}+\left[-x_{1}+2 x_{2}-2\right]^{+}\right) .
\end{aligned}
$$

By examining the different pieces of the square box $C$ separated by the three lines in the plane, we can conclude that, for $\rho>1$, the set of equilibria of the NEP $\left(C ; \theta+\rho r_{1}\right)$ is $\left\{\left(x_{1}, x_{2}\right) \mid x_{1}+x_{2}=\right.$ $\left.2,2 / 3 \leq x_{1} \leq 4 / 3\right\} \cup(2,2)$. It is clear that $(2,2)$ is not an equilibrium solution of the given GNEP $(C, D ; \theta)$.

This negative result is very informative; namely it suggests that unlike the case of an optimization problem, one needs to be selective in the penalty function in the case of the GNEP; see Theorem 5 .

(b) Squared $\ell_{2}$ penalization is not exact. The penalized problem with the squared quadratic residual function $r_{2}^{2}(x)=\left\|g^{+}(x)\right\|_{2}^{2}$ is the $\operatorname{NEP}\left(C ; \theta+\rho r_{2}^{2}\right)$. It is easy to verify that for any $\rho>0$, every point in $\left\{x \in C \mid g_{1}(x)=1 /(2 \rho), g_{2}(x) \leq 0, g_{3}(x) \leq 0\right\}$ (i.e., the red line segment in Figure 1) is an equilibrium solution of this penalized NEP. Even though as $\rho$ gets to infinity, these points converge to equilibria of the original GNEP, for any finite $\rho$ they do not belong to $C \cap D$, and thus are not equilibria of the original GNEP. 
(c) $\ell_{2}$ penalization is exact. The penalized problem with the $\ell_{2}$ residual function is the NEP $\left(C ; \theta+\rho r_{2}\right)$. By (5), at $x \notin D$, the gradient of $r_{2}(x)$ is:

$$
\nabla r_{2}(x)=\frac{1}{r_{2}(x)}\left(\begin{array}{c}
g_{1}^{+}(x)+2 g_{2}^{+}(x)-g_{3}^{+}(x) \\
g_{1}^{+}(x)-g_{2}^{+}(x)+2 g_{3}^{+}(x)
\end{array}\right)
$$

For any $x$ in the interior of $C \backslash D$, one can write down the equilibrium conditions of this NEP as

$$
\left(\begin{array}{c}
-1 \\
-1
\end{array}\right)+\rho \nabla r_{2}(x)=0
$$

which can be shown to have no solution for $\rho>1$. Similarly, by checking the respective optimality conditions of the two players' optimization problems, one can conclude that any point on the boundary of $C \backslash D$ is not be an equilibrium solution of the NEP $\left(C ; \theta+\rho r_{2}\right)$ either. Furthermore, by part (a) of Proposition 3, it is straightforward to see that any point of $C \cap D$ that is not an equilibrium solution of the $\operatorname{GNEP}(C, D ; \theta)$ cannot be one of the NEP $\left(C ; \theta+\rho r_{2}\right)$. The set of remaining feasible points of $\operatorname{NEP}\left(C ; \theta+\rho r_{2}\right)$ is the equilibria set of the $\operatorname{GNEP}(C, D ; \theta)$, i.e., $\left\{\left(x_{1}, x_{2}\right) \mid x_{1}+x_{2}=2,2 / 3 \leq x_{1} \leq 4 / 3\right\}$. Using the directional derivative formula given in (6), it is possible to verify that every point in this set is an equilibrium solution of the penalized NEP $\left(C ; \theta+\rho r_{2}\right)$.

(d) $\ell_{1}$ penalization is exact for variational equilibria. In this example, every equilibrium solution of the GNEP $(C, D ; \theta)$ is a variational equilibrium and hence a solution to the VI $(C \cap D ; \Theta)$. By penalizing the constraint set $D$ of this VI using the $\ell_{1}$ residual function (same as part (a)), we obtain the mutivalued VI $\left(C ; \Theta+\rho \partial r_{1}\right)$, where $\partial$ denotes the subdifferential of a convex function as in convex analysis. It is not difficult to see that the solution set of the penalized VI agrees with the solution set of VI $(C \cap D ; \Theta)$, and hence with the equilibrium set of the $\operatorname{GNEP}(C, D ; \theta)$, for all $\rho \geq 1$. This conclusion should be contrasted with part (a) where the penalization of this GNEP is via the NEP there. In terms of it first-order conditions, the latter NEP is equivalent to the multivalued VI $(C ; \Theta+\rho R)$, where $R$ is the multifunction $R(x)=\prod_{i=1}^{3} \partial_{x_{i}} r_{1}(x)$ which is a superset of $\partial r_{1}(x)$. Thus it is not unexpected that the NEP may have more solutions than the given GNEP, as confirmed by part (a).

(e) $\ell_{1}$ penalization is exact when $C$ is restricted. As a result of the failed exact penalization of the GNEP $(C, D ; \theta)$ in terms of the NEP $\left(C ; \theta+\rho r_{1}\right)$ demonstrated in part (a), one could raise the question of whether the given GNEP may be equivalent to a NEP $\left(\widetilde{C}, \theta+\rho r_{1}\right)$ for a certain Cartesian subset $\widetilde{C}$ of $C$. It turns out that this question has an affirmative answer for this example. Indeed, a natural way to define this restricted set is $\widetilde{C}=\widetilde{C}_{1} \times \widetilde{C}_{2}$ with

$$
\widetilde{C}_{1} \triangleq\left\{x_{1} \in C_{1} \mid \exists x_{2} \in C_{2} \text { such that }\left(x_{1}, x_{2}\right) \in D\right\}
$$

and similarly for $\widetilde{C}_{2}$. For this example, we have $\widetilde{C}_{1}=\widetilde{C}_{2}=[0,4 / 3]$. We leave it for the reader to verify that

$$
\begin{aligned}
& \text { set of equilibrium solutions of the } \operatorname{NEP}\left(\widetilde{C} ; \theta+\rho r_{1}\right) \\
= & \text { set of equilibrium solutions of the } \operatorname{GNEP}(\widetilde{C}, D ; \theta) \\
= & \text { set of equilibrium solutions of the } \operatorname{GNEP}(C, D ; \theta) .
\end{aligned}
$$


The equality between the first and second set illustrates part (a) of Proposition 3. It is possible to extend the above observation to more general cases. The formal study on this is to be pursued in the future.

An important difference between the $\ell_{1}$ and $\ell_{2}$ penalization is that the residual function in the latter case is differentiable at points outside the shared constraint set $D$ whereas that in the former is not. This observation motivates the following consideration about the choice of the residual function $r_{D}$. Namely, including the distance function to the set $D$ when the latter is closed and convex, the residual function $r_{D}$ needs to be at the minimum directionally differentiable. However, this alone is not enough as illustrated by part (a) in the above example. Thus a suitable condition on the directional derivatives outside the set $D$ is needed; such a requirement is made explicit in Theorem 5 in the next section.

\section{Main Results for the $\operatorname{GNEP}(C, D ; \theta)$}

Due to the common shared constraint set $D$, we employ a common residual function that applies to all players' individual optimization problems; see Examples 2 and 4 for such a function. Specifically, throughout this section, we let $r_{D}: \mathbb{R}^{n} \rightarrow \mathbb{R}_{+}$be a $C$-residual function of the shared constrained set $D$; i.e., for all $x \in C, r_{D}(x)=0$ if and only if $x \in D$. It then follows that for each $x^{-\nu} \in C^{-\nu}$, $r_{D}\left(\bullet, x^{-\nu}\right)$ is a $C^{\nu}$-residual function of the set $D^{\nu}\left(x^{-\nu}\right) \triangleq\left\{y^{\nu} \mid\left(y^{\nu}, x^{-\nu}\right) \in D\right\}$. Conversely, we can construct such an (aggregate) residual function $r_{D}$ from the latter sets $D^{\nu}\left(x^{-\nu}\right)$. Indeed, for each $\nu \in[N]$, let $r_{\nu}: \mathbb{R}^{n} \rightarrow \mathbb{R}_{+}$be such that for each $x^{-\nu} \in C^{-\nu}, r_{\nu}\left(\bullet, x^{-\nu}\right)$ is a $C^{\nu}$-residual

function of the set $D^{\nu}\left(x^{-\nu}\right)$. It is then easy to verify that with $r_{D}(x) \triangleq \sum_{\nu \in[N]} r_{\nu}\left(x^{\nu}, x^{-\nu}\right)$, it holds that for all $x \in C, r_{D}(x)=0$ if and only if $x \in D$. According to part (a) of Proposition 3, a key requirement for a solution of the penalized NEP $\left(C ; \theta+\rho r_{D}\right)$, where $\theta+\rho r_{D}$ is a shorthand for the vector function $\left(\theta_{\nu}+\rho r_{D}\right)_{\nu \in[N]}$, to be a solution of the $\operatorname{GNEP}(C, D ; \theta)$ is the membership of the candidate solution on hand in the shared constraint set $D$. Moreover, as mentioned above, the directional differentiability of the residual function $r_{D}$ needs to be strengthened. Taking into account these two considerations and adapting a key quantity introduced in [14, we present the following Strong Descent Assumption on residual functions. It would be shown to play a vital role in guaranteeing exact penalization of a GNEP. For a set $S \subseteq \mathbb{R}^{n}$ and a vector $x \in S$, let $\mathcal{T}(x ; S)$ denote the tangent cone at $x$ to $S$, i.e., the collection of vectors $d \in \mathbb{R}^{n}$ such that $d=\lim _{k \rightarrow \infty} \frac{x^{k}-x}{\tau_{k}}$ for some vector sequence $\left\{x^{k}\right\} \subset S$ converging to $x$ and some positive scalar sequence $\left\{\tau_{k}\right\}$ with $\tau_{k} \downarrow 0$.

Strong Descent Assumption. Let $S$ and $W$ be two closed sets and $r_{S}$ be a $W$-residual function of $S$. There exists a constant $\alpha>0$ such that for every $x \in W \backslash S, r_{S}^{\prime}(x ; d) \leq-\alpha\|d\|_{2}$ for some nonzero $d \in \mathcal{T}(x ; W)$.

The above assumption appears in [14, Theorem 3.2] in the context of a single optimization problem. While it will be discussed in some more detail momentarily, the assumption essentially requires that a strong descent direction of the residual function exists at every infeasible point $x \in W \backslash S$. Considering the optimization problem (8) with Lipschitz continuous function $f(x)$, the strong descent assumption would disqualify every point in $W \backslash S$ as a minimizer of (9) for sufficiently large $\rho$. Specialized to each player $\nu$ 's optimization problem and to the associated residual function $r_{D}\left(\bullet, x^{-\nu}\right)$, this argument leads to the next result on the exact penalization of the GNEP $(C, D ; \theta)$, where convexity of the sets $C, D$ and the functions $\theta_{\nu}\left(\bullet, x^{-\nu}\right)$ is not needed. 
Theorem 5. Suppose that $C, D$ are closed subsets of $\mathbb{R}^{n}$. Assume that each $\theta_{\nu}\left(\bullet, x^{-\nu}\right)$ is directionally differentiable and locally Lipschitz continuous with constant $\operatorname{Lip}_{\theta}>0$ on $C^{\nu}$ for all $x^{-\nu} \in C^{-\nu}$. Let $r_{D}$ be a directionally differentiable $C$-residual function of $D$. If there exist positive constants $\alpha$ and $\alpha^{\prime}$ such that for every $x \in C \backslash D$, either one of the following holds:

(a) for some $\nu \in[N]$ and nonzero $d^{\nu} \in \mathcal{T}\left(x^{\nu} ; C^{\nu}\right) \subseteq \mathbb{R}^{n_{\nu}}$,

$$
r_{D}\left(\bullet, x^{-\nu}\right)^{\prime}\left(x^{\nu} ; d^{\nu}\right) \leq-\alpha^{\prime}\left\|d^{\nu}\right\|_{2}
$$

(b) for some nonzero $d \in \mathcal{T}(x ; C)$,

$$
\sum_{\nu \in[N]} r_{D}\left(\bullet, x^{-\nu}\right)^{\prime}\left(x^{\nu} ; d^{\nu}\right) \leq r_{D}^{\prime}(x ; d) \leq-\alpha\|d\|_{2}
$$

then there exists a finite number $\bar{\rho}$ such that for every $\rho>\bar{\rho}$, every equilibrium solution of the NEP $\left(C ; \theta+\rho r_{D}\right)$ is an equilibrium solution of the $\operatorname{GNEP}(C, D ; \theta)$.

Proof. Since $\|d\|_{2} \geq \frac{1}{\sqrt{N}} \sum_{\nu \in[N]}\left\|d^{\nu}\right\|_{2}$, it follows that if (b) holds for some $\alpha$, then (a) holds with $\alpha^{\prime}=\alpha / \sqrt{N}$. Hence it suffices to prove the theorem under condition (a). Let $\bar{x}$ be an equilibrium solution of the NEP $\left(C ; \theta+\rho r_{D}\right)$. By part (a) of Proposition 3 , it suffices to show that $\bar{x} \in D$. Assume for contradiction that $\bar{x} \in C \backslash D$. By assumption, there exist $\nu \in[N]$ and $d^{\nu} \in \mathcal{T}\left(\bar{x}^{\nu} ; C^{\nu}\right)$ such that

$$
r_{D}\left(\bullet, \bar{x}^{-\nu}\right)^{\prime}\left(\bar{x}^{\nu} ; d^{\nu}\right) \leq-\alpha^{\prime}\left\|d^{\nu}\right\|_{2} .
$$

By the optimality of $\bar{x}^{\nu}$ to player $\nu$ 's optimization problem in the NEP $\left(C ; \theta+\rho r_{D}\right)$, we have

$$
\theta_{\nu}\left(\bullet, \bar{x}^{-\nu}\right)^{\prime}\left(\bar{x}^{\nu} ; d^{\nu}\right)+\rho r_{D}\left(\bullet, \bar{x}^{-\nu}\right)^{\prime}\left(\bar{x}^{\nu} ; d^{\nu}\right) \geq 0
$$

By the Lipschitz continuity of $\theta_{\nu}\left(\bullet, x^{-\nu}\right)$ with constant $\operatorname{Lip}_{\theta}$, we obtain

$$
\left|\theta_{\nu}\left(\bullet, \bar{x}^{-\nu}\right)^{\prime}\left(\bar{x}^{\nu} ; d^{\nu}\right)\right| \leq \operatorname{Lip}_{\theta}\left\|d^{\nu}\right\|_{2} .
$$

Hence, It follows that

$$
\operatorname{Lip}_{\theta}\left\|d^{\nu}\right\|_{2}-\rho \alpha^{\prime}\left\|d^{\nu}\right\|_{2} \geq 0
$$

By choosing of $\bar{\rho}=\triangleq \operatorname{Lip}_{\theta} / \alpha^{\prime}$, the above inequality yields a contradiction.

Theorem 5 is a game-theoretic extension of Theorem 1 which pertains to a single optimization problem. It is useful to note that the assumption of a Lipschitz error bound on the residual function required in the former theorem is replaced by the strong descent assumptions in the present game result. Furthermore, in contrary to part (b) of Proposition 3. Theorem 5 does not require $C^{\nu} \cap D^{\nu}\left(x^{-\nu}\right)$ to be nonempty for all $x^{-\nu} \in C^{-\nu}$. As shown in Example 4, the later nonemptiness condition may be too restrictive even for a simple linearly constrained GNEP. We also note that part (a) of Theorem 5 can be extended in a straightforward way to the general case of GNEP $(C, X ; \theta)$. The assumptions required there would involve the strong descent assumption of $r_{\nu}\left(\bullet, x^{-\nu}\right)$. While this result is not explicitly stated in the paper, another sufficient condition would be provided for the $\operatorname{GNEP}(C, X ; \theta)$ in the case that $X_{\nu}(\bullet)$ is finitely representable, see Theorem 11.

In the remaining of this section, due to the relatively simpler condition, we will focus on refining part (b) of Theorem 5 by providing some sufficient conditions for $(13)$ to hold. The inequality 
on the left side of 13 is the sum property on the total directional derivative $r_{D}^{\prime}(x ; y-x)$ with regard to the partial directional derivatives $r_{D}\left(\bullet, x^{-\nu}\right)^{\prime}\left(x^{\nu} ; y^{\nu}-x^{\nu}\right)$ for $\nu \in[N]$. The study of this relationship for a general function is originated from [2] (see also [55]). It is clear that when $r_{D}$ is convex, this inequality is equivalent to equality as the directional derivative of a convex function is subadditive in the direction. Sufficient conditions for the equality include Gâteaux differentiability, and a certain strong property of the directional derivatives that can be found in [51]. In particular, if the residual function $r_{D}$ is differentiable on $C \backslash D$ (to be precise, differentiable on a set $\Omega \backslash D$, where $\Omega$ is a open set containing the set $C)$, then the left side inequality in $(13)$ holds readily. This differentiability assumption will be in place in the rest of the section.

The inequality on the right side of $(13)$ is related to the inequality (15) imposed in Theorem 3.2 in [14] that is for the total penalization of a single optimization problem. The cited theorem dealt with the optimization problem $\underset{x \in D}{\operatorname{minimize}} \theta(x)$ where $D$ is a subset of $\mathbb{R}^{n}$ and assumed in essence to satisfy the property that there exist positive scalars $\alpha$ and $\varepsilon$ such that for all $x \in D_{\varepsilon} \backslash D$, some $y \in \mathbb{R}^{n} \backslash\{x\}$ exists satisfying $r_{D}^{\prime}(x ; y-x) \leq-\alpha\|y-x\|$, where $D_{\varepsilon}$ is an $\varepsilon$-enlargement of the set $D$; there was no second set $C$ involved. For partial penalization, the incorporation of the set $C$ in the strong descent assumption appears to be new. While this restriction reduces the set of potential descent directions from $\mathbb{R}^{n}$ to $\mathcal{T}(x ; C)$, it also excludes certain infeasible points under consideration, thus facilitating the exact penalization. We will provide a more detailed discussion on this point in subsequent subsections.

\subsection{Linear metric regularity}

The literature on Lipschitz error bounds for closed convex sets is vast. Summarizing previous papers that include [47, 43, 38] and many others, Chapter 6 in the monograph [25] provides a good reference for this theory and its applications. Closely related to Lipschitz error bounds is the theory of linear metric regularity; see [6, 45]. For our purpose, we can rephrase the strong descent assumption in terms of the latter theory. Specifically, two closed convex subsets $C$ and $D$ of $\mathbb{R}^{n}$ with a nonempty intersection are said to be linearly metrically regular if there exists a constant $\gamma^{\prime}>0$ such that

$$
\operatorname{dist}(x ; C \cap D) \leq \gamma^{\prime} \max (\operatorname{dist}(x ; C), \operatorname{dist}(x ; D)), \quad \forall x \in \mathbb{R}^{n} .
$$

We remark that linear metric regularity is equivalent to the existence of a constant $\gamma_{1}>0$ such that

$$
\operatorname{dist}(x ; C \cap D) \leq \gamma_{1} \operatorname{dist}(x ; D), \quad \forall x \in C .
$$

The above equivalence is known in the literature, e.g., see [45, Theorem 3.1]. Yet we provide a proof here for completeness. Clearly, the former implies the latter with $\gamma_{1}=\gamma$. If the latter holds, let $x \in \mathbb{R}^{n}$ be arbitrary and let $y$ be the Euclidean projection of $x$ onto $C$. We then have

$$
\begin{aligned}
\operatorname{dist}(x ; C \cap D) & \leq \operatorname{dist}(x ; C)+\operatorname{dist}(y ; C \cap D) \\
& \leq \operatorname{dist}(x ; C)+\gamma_{1} \operatorname{dist}(x ; D) \leq\left(\gamma_{1}+1\right) \max (\operatorname{dist}(x ; C), \operatorname{dist}(x ; D)),
\end{aligned}
$$

establishing the equivalence of linear metric regularity and the inequality (14). It follows from this equivalence that if $C$ and $D$ are linearly metrically regular, then a $C$-residual function $r_{D}$ of the set $D$ satisfies a $C$-Lipschitz error bound if there exists a constant $\gamma>0$ such that $\operatorname{dist}(x ; D) \leq \gamma r_{D}(x)$ for all $x \in C$, the latter condition being a usual error bound for $D$ with reference to vectors in $C$.

The result below shows that linear metric regularity (or equivalently (14)) provides a sufficient condition for the strong descent assumption to hold. 
Proposition 6. Suppose the two closed convex sets $C$ and $D$ with nonempty intersection are linearly metrically regular with constant $\gamma^{\prime}$. Let $r_{D}$ be a convex residual function of $D$ satisfying a $C$-Lipschitz error bound for $D$ with constant $\gamma>0$. If $r_{D}$ is differentiable on the set $C \backslash D$, then (13) holds.

Proof. It remains to show the right side of 113 holds with $\alpha=\frac{1}{\gamma \gamma^{\prime}}$. Let $x \in C \backslash D$ and $y$ be the Euclidean projection of $x$ onto the intersection $C \cap D$. By the convexity of the residual function $r_{D}$, we have

$$
\begin{aligned}
r_{D}^{\prime}(x ; y-x) & \leq r_{D}(y)-r_{D}(x)=-r_{D}(x) \leq-\gamma \operatorname{dist}(x ; D) \\
& \leq-\frac{\gamma}{\gamma^{\prime}} \operatorname{dist}(x ; C \cap D)=-\frac{\gamma}{\gamma^{\prime}}\|x-y\|_{2}
\end{aligned}
$$

as desired.

In addition to the necessary and sufficient inequality (14), there are well-known sufficient conditions for two closed convex sets to be linearly metrically regular. In particular, we provide in Proposition 7 below a Slater condition (part (a)) and a polyhedrality condition (part (b)). The proof of the first statement of the proposition follows from [6, Corollary 5, Remark 9 and 10] and and that of the second statement from the well-known Hoffman error bound for polyhedra [25, Lemma 3.2.3]. In the result, we use the shorthand "rint" to denote the relative interior of a convex set.

Proposition 7. Two closed convex sets $C$ and $D$ in $\mathbb{R}^{n}$ are linearly metrically regular under either one of the following two conditions:

(a) $C \cap D$ is bounded and $\operatorname{rint}(C) \cap \operatorname{rint}(D) \neq \emptyset$;

(b) $C$ and $D$ are both polyhedra.

Combining part (b) of Theorem 5 and Propositions 6 and 7 , we have the following exact penalization result for the $\operatorname{GNEP}(C, D ; \theta)$ in terms of the penalized NEP $\left(C, \theta+\rho r_{D}\right)$, for which no proof is needed.

Corollary 8. Let $D$ be a closed convex set in $\mathbb{R}^{n}$ and $C \triangleq \prod_{\nu \in[N]} C^{\nu}$ where each $C^{\nu}$ is a closed convex subset of $\mathbb{R}^{n_{\nu}}$. Assume that each $\theta_{\nu}\left(\bullet, x^{-\nu}\right)$ is convex and locally Lipschitz continuous on the set $C^{\nu}$ with constant $\operatorname{Lip}_{\theta}>0$ for all $x^{-\nu} \in C^{-\nu}$. Let $r_{D}$ be a convex $C$-residual function of the set $D$ that is differentiable on $C \backslash D$. Then under either one of the following two conditions, there exists a scalar $\bar{\rho}>0$ such that for all $\rho>\bar{\rho}$, every equilibrium solution of the NEP $\left(C ; \theta+\rho r_{D}\right)$ is an equilibrium solution of the $\operatorname{GNEP}(C, D ; \theta)$ :

(a) $r_{D}$ satisfies a $C$-Lipschitz error bound for the set $D ; C \cap D$ is bounded; and $\operatorname{rint}(C) \cap \operatorname{rint}(D)$ is nonempty;

(b) $C$ and $D$ are both polyhedra.

Part (b) of Corollary 8 provides a formal generalization of part (c) of the illustrative Example 4 It also demonstrates that, for shared linearly constrained games and with the use of $\ell_{q}$ residual functions for $q \in(1, \infty)$, the Lipschitz error bound condition required in part (a) is no longer needed. In the next subsection, we will show that this is true in general for convex finitely representable set $D$. 


\subsection{Shared finitely representable sets}

In this subsection and the rest of the paper, we assume that the set $C$ is compact and convex. We discuss the GNEP $(C, D ; \theta)$ where the shared-constrained set $D$ is finitely representable by coupled differentiable inequalities and linear equations. Specifically, let

$$
D \triangleq\left\{x \in \mathbb{R}^{n} \mid g(x) \leq 0 \text { and } h(x)=0\right\}
$$

with $h(x)=A x-b$ for some matrix $A \in \mathbb{R}^{p}$ and vector $b \in \mathbb{R}^{p}$ and each $g_{j}: \mathbb{R}^{n} \rightarrow \mathbb{R}$ for $j \in[m]$ being convex and differentiable. We separate the linear and nonlinear constraints in $D$ so that we can state the Slater condition more precisely; see assumption in Lemma 9. We employ the $\ell_{q}$ residual function for an arbitrary $q \in(1, \infty)$ for the set $D$ :

$$
r_{q}(x) \triangleq\left\|\left(\begin{array}{c}
\max (g(x), 0) \\
h(x)
\end{array}\right)\right\|_{q}, \quad x \in \mathbb{R}^{n} .
$$

The function $r_{q}$ is continuously differentiable on the complement of the set $D$. Toward the formulation of an exact penaliztion result for the shared constrained GNEP $(C, D ; \theta)$ with a finitely representable set $D$, we establish a consequence of the Slater CQ for the set $D$. This lemma is related to many results in the literature; but surprisingly, we cannot find a source that has this exact result. As such, we give a detailed proof.

Lemma 9. In the above setting, let $C$ be a compact convex set in $\mathbb{R}^{n}$. Suppose that there exists a vector $\bar{x} \in \operatorname{rint} C$ such that $A \bar{x}=b$ and $g(\bar{x})<0$. Then there exists a scalar $\alpha>0$ such that for every $x \in C \backslash D$, a vector $\widehat{x} \in C$ exists such that $r_{q}^{\prime}(x ; \widehat{x}-x) \leq-\alpha\|\widehat{x}-x\|_{2}$.

Proof. Let $\varepsilon>0$ be such that the closed set $\mathbb{B}_{\varepsilon}(\bar{x}) \triangleq\left\{x \in C \mid\|x-\bar{x}\|_{2} \leq \varepsilon\right\}$ is contained in $\{x \in C \mid g(x)<0\}$. That such a scalar $\varepsilon$ exists is by the property of the Slater point $\bar{x}$. Let $\Sigma$ be the family of tuples $\sigma \in\{0,1,-1\}^{p}$ such that there exists $x \in C$ satisfying sgn $(h(x))=\sigma$, where sgn $(h(x))$ is the $p$-vector whose $i$ th component is equal to the sign of $h_{i}(x)$ if $h_{i}(x) \neq 0$ and equal to 0 otherwise. For each $\sigma \in \Sigma$, let

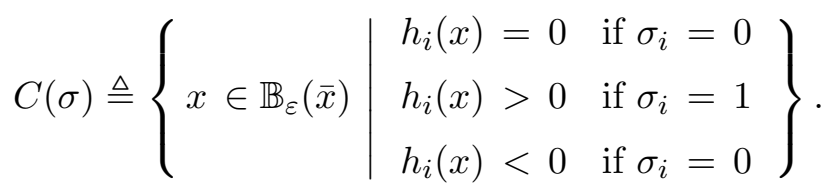

This set $C(\sigma)$ must be nonempty because for any $\sigma \in \Sigma$, letting $x^{\sigma}$ be a vector in $C$ such that $\operatorname{sgn}\left(h\left(x^{\sigma}\right)\right)=\sigma$, by the linearlity of $h$ and the fact that $h(\bar{x})=0$, we have

$$
\operatorname{sgn}\left(h\left(\bar{x}+\tau\left(x^{\sigma}-\bar{x}\right)\right)\right)=\operatorname{sgn}\left(h\left(x^{\sigma}\right)\right)=\sigma,
$$

thus $\bar{x}+\tau\left(x^{\sigma}-\bar{x}\right) \in C(\sigma)$ for all $\tau \in(0,1]$ sufficiently small. Furthermore, since $C$ is convex and $\bar{x} \in \operatorname{rint} C$, for some $\tau<0$ that is sufficiently close to $0, \bar{x}+\tau\left(x^{\sigma}-\bar{x}\right) \in C$ and $\operatorname{sgn}\left(h\left(\bar{x}+\tau\left(x^{\sigma}-\right.\right.\right.$ $\bar{x})))=-\sigma$. Therefore, $\Sigma=-\Sigma$ and for every $x \in C,-\operatorname{sgn} h(x) \in \Sigma$. For each $\sigma \in \Sigma$, define

$$
\mu(\sigma) \triangleq \begin{cases}1 & \text { if } \sigma=0 \\ \max _{x \in \operatorname{cl} C(\sigma)}\left[\min _{i: \sigma_{i} \neq 0} \sigma_{i} h_{i}(x)\right] & \text { if } \sigma \neq 0 .\end{cases}
$$


where $\mathrm{cl}$ denotes the closure of a set. Since $\min _{i: \sigma_{i} \neq 0} \sigma_{i} h_{i}(x)$ is a continuous function of $x$ and $\operatorname{cl} C(\sigma)$ is compact, it follows that maximum in $\mu(\sigma)$ is attained if $\sigma \neq 0$. Hence $\mu(\sigma)$ is a positive scalar for all $\sigma \in \Sigma$. Let

$$
y(\sigma) \begin{cases}\triangleq \bar{x} & \text { if } \sigma=0 \\ \in \operatorname{lacl}_{x \in \operatorname{cl}(\sigma)}^{\operatorname{argmax}}\left[\min _{i: \sigma_{i} \neq 0} \sigma_{i} h_{i}(x)\right] & \text { if } \sigma \neq 0 .\end{cases}
$$

Note that $g(y(\sigma))<0$ for all $\sigma \in \Sigma$. Now define the scalar

$$
\eta \triangleq \min _{\sigma \in \Sigma}\left\{\mu(\sigma), \min _{i \in[m]}\left[-g_{i}(y(\sigma))\right]\right\}
$$

which must be positive. For $x \in C \backslash D$, sgn $(h(x))$ is a nonzero sign tuple in the family $\Xi$. Let $\widehat{x} \triangleq y(-\operatorname{sgn}(h(x)))$. By the gradient inequality of convex functions and the definition of $\eta$, we have the following:

$$
\begin{array}{cl}
\nabla g_{i}(x)^{\mathrm{T}}(\widehat{x}-x) & \leq g_{i}(\widehat{x})=g_{i}(y(-\operatorname{sgn} h(x))) \leq-\eta \text { if } g_{i}(x)>0 \\
\left(\operatorname{sgn} h_{j}(x)\right) \nabla h_{j}(x)^{T}(\widehat{x}-x) & \leq\left(\operatorname{sgn} h_{j}(x)\right) h_{j}(y(-\operatorname{sgn} h(x))) \leq-\eta \text { if } h_{j}(x) \neq 0 .
\end{array}
$$

By the gradient formula (5), we deduce,

$$
\begin{aligned}
\nabla r_{q}(x)^{T}(\widehat{x}-x) & =\frac{1}{\left(r_{q}(x)\right)^{q-1}}\left[\sum_{i \in[m]}\left[\max \left(g_{i}(x), 0\right)\right]^{q-1} \nabla_{x^{\nu}} g_{i}(x)+\left.h_{j}(x)\right|^{q-1}\left(\operatorname{sgn} h_{j}(x)\right) \nabla_{x^{\nu}} h_{j}(x)\right]^{T}(\widehat{x}-x) \\
& \leq-\eta\left(\frac{r_{q-1}(x)}{r_{q}(x)}\right)^{q-1} \leq-\eta c_{q}^{q-1} \leq-\alpha\|\widehat{x}-x\|_{2},
\end{aligned}
$$

where $c_{q}>0$ is the constant such that $c_{q}\|\bullet\|_{q} \leq\|\bullet\|_{q-1}$ and $\alpha \triangleq \frac{\eta c_{q}^{q-1}}{2 \max _{x \in C}\|x\|_{2}}$.

The following exact penalization result for the shared-constrained GNEP is an immediate consequence of the above lemma and part (b) of Theorem 5. There is no need for a proof.

Theorem 10. Let each $C^{\nu}$ be a compact convex subset of $\mathbb{R}^{n_{\nu}}$ and $D$ be given by (15) with $h(x)=A x-b$ being affine and each $g_{j}(\bullet)$ being convex and differentiable for all $x^{-\nu} \in C^{-\nu}$. Assume that each $\theta_{\nu}\left(\bullet, x^{-\nu}\right)$ is convex and Lipschitz continuous on the set $C^{\nu}$ with constant $\operatorname{Lip}_{\theta}>0$ for all $x^{-\nu} \in C^{-\nu}$. Let $q \in(1, \infty)$ be given. The following two statements hold.

(a) For every $\rho>0$, the $\operatorname{NEP}\left(C ; \theta+\rho r_{q}\right)$ has an equilibrium solution.

(b) Under the assumption of Lemma $9, \bar{\rho}>0$ exists such that for every $\rho>\bar{\rho}$, every equilibrium solution of the $\operatorname{NEP}\left(C ; \theta+\rho r_{q}\right)$ is a equilibrium solution of the $\operatorname{GNEP}(C, D ; \theta)$.

We note that the property of boundedness and convexity of the sets $C^{\nu}$ is used to assert the existence of a solution to the penalized NEP for any $\rho>0$. Without such existence, the result is not meaningful, unless we have a converse in place to argue that the GNEP will not have a solution in this case. This is one aspect that we have de-emphasized in the discussion. 
As a final remark of this section, we note that by following similar arguments, we can establish an exact $\ell_{1}$-penalty result for the variational equilibria of the shared-constrained GNEP $(C, D ; \theta)$ with a convex, finitely representable set $D$ under the standard Slater condition. The proof would use the directional derivative formula (7) for this penalty function instead of the gradient formula (5). The details are omitted. Such a result provides a generalization of part (d) of the illustrative Example 4.

\section{$5 \quad$ Non-Shared Finitely Representable Sets}

In spite of the considerable amount of literature on the exact penalization of a GNEP with coupled finitely representable constraints, most existing results pertain to problems with only inequality constraints, see e.g., [23, 24, 31, 36]. The reason for the absence of such an extended treatment to allow for equality constraints (even linear ones) is not exactly clear; one possible reason might be that the EMFCQ used in the aforementioned papers is not easily amenable to generalization to handle equality constraints. In this section, we extend the discussion in Subsection 4.2 to the GNEP $(C, X ; \theta)$ with non-shared coupled finitely representable constraints and show that an extended Slater-type CQ can be imposed to yield the exact penalization for such a GNEP. Specifically, we let the sets $X^{\nu}\left(x^{-\nu}\right)$ be defined as follows: for each $x^{-\nu} \in C^{-\nu}$,

$$
X^{\nu}\left(x^{-\nu}\right) \triangleq\left\{x^{\nu} \in \mathbb{R}^{n_{\nu}} \mid g^{\nu}\left(x^{\nu}, x^{-\nu}\right) \leq 0 \text { and } h^{\nu}(x)=0\right\}
$$

with each $h^{\nu}: \mathbb{R}^{n} \rightarrow \mathbb{R}^{p_{\nu}}$ being an affine function and each $g_{j}^{\nu}: \mathbb{R}^{n} \rightarrow \mathbb{R}$ for $j \in\left[m_{\nu}\right]$ being such that $g_{j}^{\nu}\left(\bullet, x^{-\nu}\right)$ is convex and differentiable for every $x^{-\nu} \in C^{-\nu}$. We present a result that is a parallel of Theorem 10 for $\operatorname{GNEP}(C, X ; \theta)$ and then we connect the assumptions used in the result with the commonly used EMFCQ. Let

$$
r_{\nu ; q}(x) \triangleq\left\|\left(\begin{array}{c}
\max \left(g^{\nu}(x), 0\right) \\
h^{\nu}(x)
\end{array}\right)\right\|_{q}, \quad x \in \mathbb{R}^{n}
$$

be the $\ell_{q}$ residual function for the set $X^{\nu}\left(x^{-\nu}\right)$. This function $r_{\nu ; q}\left(\bullet, x^{-\nu}\right)$ is continuously differentiable on the complement of the set $X^{\nu}\left(x^{-\nu}\right)$ for all $x^{-\nu}$. Let $r_{X ; q} \triangleq\left(r_{\nu ; q}\right)_{\nu \in[N]}$. The penalized Nash game, NEP $\left(C ; \theta+\rho r_{X ; q}\right)$, consists of the following family of optimization problems:

$$
\left\{\operatorname{minimize}_{x^{\nu} \in C^{\nu}} \theta_{\nu}\left(x^{\nu}, x^{-\nu}\right)+\rho r_{\nu ; q}\left(x^{\nu}, x^{-\nu}\right)\right\}_{\nu=1}^{N},
$$

for a given penalty parameter $\rho>0$.

Theorem 11. Let each $C^{\nu}$ be a compact convex subset of $\mathbb{R}^{n_{\nu}}$. Let each $X^{\nu}$ be defined as in (16) with $h^{\nu}$ being affine and each $g_{j}^{\nu}\left(\bullet, x^{-\nu}\right)$ be convex and differentiable for all $x^{-\nu} \in C^{-\nu}$. Assume that each $\theta_{\nu}\left(\bullet, x^{-\nu}\right)$ is convex and Lipschitz continuous on the set $C^{\nu}$ with constant $\operatorname{Lip}_{\theta}>0$ for all $x^{-\nu} \in C^{-\nu}$. Let $q \in(1, \infty)$ be given. The following three statements hold.

(a) For every $\rho>0$, the NEP $\left(C ; \theta+\rho r_{X ; q}\right)$ has an equilibrium solution.

(b) If there exists $\alpha>0$ such that for every $x \in C$ not in the graph of the multifunction $X$, there exists $\widehat{x} \in C$ satisfying for some $\widehat{\nu} \in[N]$ with $x^{\widehat{\nu}} \notin X^{\widehat{\nu}}\left(x^{-\widehat{\nu}}\right)$,

- for all $i \in\left[m_{\nu}\right], \nabla_{x^{\widehat{\nu}}} g_{i}^{\widehat{\nu}}\left(x^{\widehat{\nu}}, x^{-\widehat{\nu}}\right)^{T}\left(\widehat{x} \widehat{\nu}-x^{\widehat{\nu}}\right) \leq-\alpha$ if $g_{i}^{\widehat{\nu}}(x)>0$, and 
- for all $j \in\left[p_{\nu}\right],\left[\operatorname{sgn} h_{j}^{\widehat{\nu}}(x)\right] \nabla_{x^{\widehat{\nu}}} h_{j}^{\widehat{\nu}}\left(x^{\widehat{\nu}}, x^{-\widehat{\nu}}\right)^{T}\left(\widehat{x}^{\widehat{\nu}}-x^{\widehat{\nu}}\right) \leq-\alpha$ if $h_{j}^{\widehat{\nu}}(x) \neq 0$,

then $\bar{\rho}>0$ exists such that for every $\rho>\bar{\rho}$, every equilibrium solution of the $\operatorname{NEP}\left(C ; \theta+\rho r_{X ; q}\right)$ is a equilibrium solution of the $\operatorname{GNEP}(C, X ; \theta)$.

Proof. Assertion (a) follows from a standard existence result for a NEP. In order to prove statement (b), let $x^{*}$ be an equilibrium solution of the $\operatorname{NEP}\left(C ; \theta+\rho r_{X ; q}\right)$ for some $\rho>0$ which will be specified momentarily. By part (a) of Proposition 3 , it suffices to show that $x^{*}$ belongs to the graph of the multifunction $X$. Assume the contrary; let $\widehat{x}$ and $\widehat{\nu}$ be the vector and player label, respectively, associated with $x^{*}$ as stipulated by the assumption in this part. By the gradient formula (5), we deduce,

$$
\begin{aligned}
& \nabla_{x^{\widehat{\nu}}} r_{\widehat{\nu} ; q}\left(x^{*}\right)^{T}\left(\widehat{x}^{\widehat{\nu}}-x^{*, \widehat{\nu}}\right) \\
& \left.=\frac{1}{r_{\widehat{\nu} ; q}^{q-1}\left(x^{*}\right)}\left[\sum_{i \in\left[m_{\widehat{\nu}]}\right.}\left[\max \left(g_{i}^{\widehat{\nu}}\left(x^{*}\right), 0\right)\right]^{q-1} \nabla_{x^{\widehat{\nu}}} g_{i}^{\widehat{\nu}}\left(x^{*}\right)+\right]_{j \in\left[p_{\widehat{\nu}}\right]}\left|h_{j}^{\widehat{\nu}}\left(x^{*}\right)\right|^{q-1}\left(\operatorname{sgn} h_{j}^{\widehat{\nu}}\left(x^{*}\right)\right) \nabla_{x^{\widehat{\nu}}} h_{j}^{\widehat{\nu}}\left(x^{*}\right)\right]^{T}\left(\widehat{x}^{\widehat{\nu}}-x^{*, \widehat{\nu}}\right) \\
& \leq-\alpha\left(\frac{r_{\widehat{\nu}, q-1}\left(x^{*}\right)}{r_{\widehat{\nu}, q}\left(x^{*}\right)}\right)^{q-1} \leq-\alpha c_{q}^{q-1},
\end{aligned}
$$

where $c_{q}>0$ is the constant such that $c_{q}\|\bullet\|_{q} \leq\|\bullet\|_{q-1}$. Since

$$
x^{*, \widehat{\nu}} \in \underset{x^{\widehat{\nu}} \in C^{\widehat{\nu}} \cap X^{\widehat{\nu}}\left(x^{*,-\widehat{\nu}}\right)}{\operatorname{minimize}} \theta_{\widehat{\nu}}\left(x^{\widehat{\nu}}, x^{*,-\widehat{\nu}}\right)+\rho r_{\widehat{\nu} ; q}\left(x^{\widehat{\nu}}, x^{*,-\widehat{\nu}}\right),
$$

we deduce

$$
\begin{aligned}
0 & \leq \theta_{\widehat{\nu}}\left(\bullet, x^{*,-\widehat{\nu}}\right)^{\prime}\left(x^{*, \widehat{\nu}} ; \widehat{x}^{\widehat{\nu}}-x^{*, \widehat{\nu}}\right)+\rho \nabla_{x^{\widehat{\nu}}} r_{\widehat{\nu} ; q}\left(x^{*}\right)^{T}\left(\widehat{x}^{\widehat{\nu}}-x^{*, \widehat{\nu}}\right) \\
& \leq \operatorname{Lip}_{\theta}\left\|\widehat{x}^{\widehat{\nu}}-x^{*, \widehat{\nu}}\right\|_{2}-\rho \alpha c_{q}^{q-1} \leq\left[\operatorname{Lip}_{\theta}-\rho \widehat{\alpha}\right]\left\|\widehat{x}^{\widehat{\nu}}-x^{*, \widehat{\nu}}\right\|_{2} .
\end{aligned}
$$

where $\widehat{\alpha} \triangleq \frac{\alpha c_{q}^{q-1}}{2 \max _{x \in C}\|x\|_{2}}$. Provided that $\rho>\widehat{\alpha}$, the above inequality yields a contradiction.

We close this paper by showing that the commonly used EMFCQ is a more restrictive assumption than the one imposed in part (b) of Theorem 11. Besides the fact that the EMFCQ does not admit equality constraints, there is another subtle restriction in it; namely, included in this CQ is a condition on a feasible vector in the graph of the multifunction $X$, whereas the condition in part (b) of Theorem 11 does not contain such a restriction. We state the EMFCQ for a convex set $C$ as follows, see also [12, Definition 4.3].

(EMFCQ) This CQ is said to hold for the $\operatorname{GNEP}(C, X ; \theta)$, where each

$$
X^{\nu}\left(x^{-\nu}\right)=\left\{x^{\nu} \mid g^{\nu}\left(x^{\nu}, x^{-\nu}\right) \leq 0\right\}
$$

with each $g_{i}^{\nu}\left(\bullet, x^{-\nu}\right)$ being convex and continuously differentiable, if for every $x \in C$ and $\nu \in[N]$, there exists a vector $\widehat{x} \in C$ such that

$$
\nabla_{x^{\nu}} g_{i}^{\nu}\left(x^{\nu}, x^{-\nu}\right)^{T}\left(\widehat{x}^{\nu}-x^{\nu}\right)<0 \quad \text { if } g_{i}^{\nu}\left(x^{\nu}, x^{-\nu}\right) \geq 0 .
$$

Note that this CQ restricts even a vector $x \in C$ belonging to the graph of the multifunction $X$, i.e., even for a feasible vector $x$ to the GNEP. The reason to include such vectors in the CQ is so 
that the Karush-Kuhn-Tucker conditions become valid if $x$ turns out be an equilibrium solution of the GNEP. It turns out that as far as exact penalization is concerned, the validity of the CQ can be somewhat relaxed. We have the following result.

Proposition 12. Let each $C^{\nu}$ be a convex compact subset of $\mathbb{R}^{n_{\nu}}$ and each $X^{\nu}$ be defined as in (18) with each $g_{i}^{\nu}\left(\bullet, x^{-\nu}\right)$ being convex and continuously differentiable. If EMFCQ holds for the $\operatorname{GNEP}(C, X ; \theta)$, then the assumption in part (b) of Theorem is valid.

Proof. The proof is by contradiction. We first restate the assumption in part (b) in Theorem 11 in a set-theoretic form that is more conducive to obtain the contradiction. For a given $x \in C$, let

$$
\mathcal{I}_{\nu}(x) \triangleq\left\{i \in\left\{1, \cdots, m_{\nu}\right\} \mid g_{i}^{\nu}\left(x^{\nu}, x^{-\nu}\right)>0\right\}
$$

be the index set of violated constraints in $X^{\nu}\left(x^{-\nu}\right)$ by $x^{\nu}$. The statement that $x^{\nu} \notin X^{\nu}\left(x^{-\nu}\right)$ is equivalent to $\mathcal{I}_{\nu}(x) \neq \emptyset$. Also let

$$
\widehat{\mathcal{I}}(x) \triangleq\left\{\nu \in[N] \mid \mathcal{I}_{\nu}(x) \neq \emptyset\right\}
$$

be the player labels $\nu$ for which there is at least one constraint in $X^{\nu}\left(x^{-\nu}\right)$ that is not satisfied by $x^{\nu}$. Thus $x \notin \operatorname{graph}(X)$ if and only if $\widehat{\mathcal{I}}(x) \neq \emptyset$. Coupled with a $y \in C$, define the quantity

$$
\alpha_{\nu}(x, y) \triangleq \max _{i \in \mathcal{I}_{\nu}(x)} \nabla_{x^{\nu}} g_{i}^{\nu}\left(x^{\nu}, x^{-\nu}\right)^{T}\left(y^{\nu}-x^{\nu}\right)
$$

By convention, the maximum over the empty set is equal to $-\infty$. For a given vector $x \in C$ not in $\operatorname{graph}(X)$ and a player label $\nu \in \widehat{\mathcal{I}}(x)$, the quantity $\alpha_{\nu}(x, \bullet)$ is a continuous function on $C$. Thus it is justified to define the scalar

$$
\gamma \triangleq \sup _{x \in C \backslash \operatorname{graph}(X)}\left[\min _{\nu \in \widehat{\mathcal{I}}(x)}\left(\min _{y \in C} \alpha_{\nu}(x, y)\right)\right],
$$

where we use sup instead of max because the latter may not be attained. Now, the assumption in part (b) in Theorem 11 becomes $\gamma<0$. So to prove the proposition by contradiction, suppose $\gamma \geq 0$. Then there exist a sequence of vectors $\left\{x^{k}\right\} \subset C$ and a sequence of positive scalars $\left\{\varepsilon_{k}\right\} \downarrow 0$ such that for every $k, x^{k} \notin \operatorname{graph}(X)$ and there exists $\nu_{k} \in \widehat{\mathcal{I}}\left(x^{k}\right)$ such that $\min _{y \in C} \alpha_{\nu_{k}}\left(x^{k}, y\right) \geq-\varepsilon_{k}$. Without loss of generality, we may assume that the sequence $\left\{x^{k}\right\}$ converges to a vector $x^{\infty} \in C$. Since there are only finitely many players, it follows that there exists a subsequence $\left\{x^{k}\right\}_{k \in \kappa}$ and a player label $\bar{\nu}$ such that for all $k \in \kappa$, we have $\bar{\nu} \in \widehat{\mathcal{I}}\left(x^{k}\right)$ and $\min _{y \in C} \alpha_{\bar{\nu}}\left(x^{k}, y\right) \geq-\varepsilon_{k}$. Hence for all $y \in C, \alpha_{\bar{\nu}}\left(x^{k}, y\right) \geq-\varepsilon_{k}$ for all $k \in \kappa$. Passing to the limit $k(\in \kappa) \rightarrow \infty$, we deduce that $\min _{y \in C} \alpha_{\bar{\nu}}\left(x^{\infty}, y\right) \geq 0$. Since $\bar{\nu} \in \widehat{\mathcal{I}}\left(x^{k}\right)$ for all $k \in \kappa$, it follows that $g_{i}^{\bar{\nu}}\left(x^{\infty}\right) \geq 0$ for all $i \in \mathcal{I}_{\bar{\nu}}\left(x^{\infty}\right)$. Thus we have obtained the following

$$
\forall y \in C \exists i \text { such that } g_{i}^{\bar{\nu}}\left(x^{\infty}\right) \geq 0 \text { and } \nabla_{x^{\nu}} g_{i}^{\bar{\nu}}\left(x^{\infty, \bar{\nu}}, x^{\infty,-\bar{\nu}}\right)^{T}\left(y^{\bar{\nu}}-x^{\infty, \bar{\nu}}\right) \geq 0 .
$$

But this contradicts the EMFCQ applied to $x^{\infty}$ which asserts that there exists $\widehat{x} \in C$ such that $\nabla_{x^{\nu}} g_{i}^{\nu}\left(x^{\infty, \nu}, x^{\infty,-\nu}\right)^{T}\left(\widehat{x}^{\nu}-x^{\infty, \nu}\right)<0$ whenever $g_{i}^{\nu}\left(x^{\infty, \nu}, x^{\infty,-\nu}\right) \geq 0$.

Conclusion. In this paper, we have provided a comprehensive exact penalization theory for the GNEP, by putting together a systematic study of the subject that begins with an illustrative example and ends with a pair of results for the cases of finitely representable constraint sets. In the 
process, we have clarified the role of error bounds and constraint qualifications in the developed theory; this is something that has not been sufficiently emphasized in the existing literature of the GNEP. How these exact penalization results can be put to use in the development of improved algorithms for computing an equilibrium solution of the GNEP remains to be investigated.

Acknowledgement. The authors are grateful to Christian Kanzow and Francisco Facchinei for some helpful comments on a draft of this paper.

\section{References}

[1] Kenneth J. Arrow and Gerard Debreu. Existence of an equilibrium for a competitive economy. Econometrica: Journal of the Econometric Society 22(3): 265-290, 1954.

[2] Alfred Auslender. Optimisation Méthodes Numériques. Masson, Paris, France, 1976.

[3] Didier Aussel and Simone Sagratella Sufficient conditions to compute any solution of a quasivariational inequality via a variational inequality. Mathematical Methods of Operations Research 85(1), 3-18, 2017.

[4] Jeff X. Ban, Jong-Shi Pang, and Maged Dessouky. A general equilibrium model for transportation systems with e-hailing services and flow congestion. Manuscript, Department of Industrial and Systems Engineering, University of Southern California. September 2018.

[5] A Bassanini, A La Bella, and A Nastasi. Allocation of railroad capacity under competition: a game theoretic approach to track time pricing. In Transportation and Network Analysis: Current Trends, pages 1-17. Springer, 2002.

[6] Heinz H. Bauschke, Jonathan M. Borwein, and Wu Li. Strong conical hull intersection property, bounded linear regularity, Jameson's property $(\mathrm{g})$, and error bounds in convex optimization. Mathematical Programming 86(1):135-160, 1999.

[7] Michele Breton, Georges Zaccour, and Mehdi Zahaf. A game-theoretic formulation of joint implementation of environmental projects. European Journal of Operational Research 168(1):221-239, 2006.

[8] Richard H. Byrd, Jorge Nocedal, and Richard A. Waltz. Steering exact penalty methods for nonlinear programming. Optimization Methods and Software 23(2): 197-213, 2008.

[9] Richard H. Byrd, G Lopez-Calva, and Jorge Nocedal. A line search exact penalty method using steering rules. Mathematical Programming 133(1-2): 39-73, 2012.

[10] Richard H. Byrd, Jorge Nocedal, and Richard A. Waltz. Knitro: An integrated package for nonlinear optimization. In G. Di Pillo and M. Roma, editors. Large-Scale Nonlinear Optimization, pages 35-59, Springer, Boston, 2006.

[11] Francis H. Clarke. Optimization and Nonsmooth Analysis. Classics in Applied Mathematics, Volume 5, Society for Industrial and Applied Mathematics, 1990. [Reprint from John Wiley Publishers, New York 1983.]

[12] Roberto Cominetti, Francisco Facchinei and Jean-Bernard Lasserre. Authors. Modern Optimization Modeling Techniques. Birkhauser Springer Basel, 2012. 10.1007/978-3-0348-0291-8. 
[13] Gerard Debreu. A social equilibrium existence theorem. Proceedings of the National Academy of Sciences 38(10):886-893, 1952.

[14] Vladimir F. Demyanov, Gianni Di Pillo, and Francisco Facchinei. Exact penalization via Dini and Hadamard conditional derivatives. Optimization Methods and Software 9(1-3):19$36,1998$.

[15] Gianni Di Pillo and F Facchinei. Exact penalty functions for nondifferentiable programming problems. In Nonsmooth optimization and related topics, pages 89-107. Springer, 1989.

[16] Gianni Di Pillo and Francisco Facchinei. Regularity conditions and exact penalty functions in Lipschitz programming problems. Nonsmooth optimization methods and applications, pages 107-120, 1992.

[17] Gianni Di Pillo and Francisco Facchinei. Exact barrier function methods for Lipschitz programs. Applied Mathematics and Optimization 32(1):1-31, 1995.

[18] G. Di Pillo and L. Grippo Exact penalty functions in constrained optimization. SIAM Journal on control and optimization 27(6), 1333-1360, 1989.

[19] Axel Dreves. Computing all solutions of linear generalized Nash equilibrium problems. Mathematical Methods of Operations Research 85(2):207-221, 2017.

[20] Axel Dreves, Francisco Facchinei, Christian Kanzow, and Simone Sagratella. On the solution of the KKT conditions of generalized Nash equilibrium problems. SIAM Journal on Optimization 21(3):1082-1108, 2011.

[21] Francisco Facchinei, Andreas Fischer, and Veronica Piccialli. On generalized Nash games and variational inequalities. Operations Research Letters 35:159-164, 2007.

[22] Francisco Facchinei and Christian Kanzow. Generalized Nash equilibrium problems. Annals of Operations Research 175(1):177-211, 2007.

[23] Francisco Facchinei and Christian Kanzow. Penalty methods for the solution of generalized Nash equilibrium problems. SIAM Journal on Optimization 20(5):2228-2253, 2010.

[24] Facchinei Facchinei and Lorenzo Lampariello. Partial penalization for the solution of generalized Nash equilibrium problems. Journal of Global Optimization 50(1): 39-50, 2011.

[25] Francisco Facchinei and Jong-Shi Pang. Finite-dimensional variational inequalities and complementarity problems. Springer Science \& Business Media, 2003.

[26] Francisco Facchinei and Jong-Shi Pang. Exact penalty functions for generalized Nash problems. In Large-scale nonlinear optimization, pages 115-126. Springer, 2006.

[27] Francisco Facchinei and Jong-Shi Pang. Nash equilibria: the variational approach. Chapter 12 in Daniel P. Palomar and Yonica C. Eldar, editors. Convex optimization in signal processing and communications, Cambridge University Press, pages 443-493, 2010.

[28] Francisco Facchinei and Simone Sagratella. On the computation of all solutions of jointly convex generalized Nash equilibrium problems. Optimization Letters, 5(3):531-547, 2011. 
[29] Anthony V. Fiacco and Garth P. McCormick. Nonlinear Programming: Sequential Unconstrained Minimization Techniques. Classics in Applied Mathematics, Volume 4, Society for Industrial and Applied Mathematics, 1990. [Reprint from John Wiley Publishers, New York 1968.]

[30] Andreas Fischer, Markus Herrich, and Klaus Schönefeld. Generalized Nash equilibrium problems-recent advances and challenges. Pesquisa Operacional, 34(3):521-558, 2014.

[31] Masao Fukushima. Restricted generalized Nash equilibria and controlled penalty algorithm. Computational Management Science, 8(3):201-218, 2011.

[32] J Gwinner. On the penalty method for constrained variational inequalities. Optimization: Theory and Algorithms 86(1): 197-211, 1983.

[33] S.P. Han and O.L. Mangasarian. Exact penalty functions in nonlinear programming. Mathematical Programming 17(1): 251-269, 1979.

[34] Patrick T Harker. Generalized Nash games and quasi-variational inequalities. European Journal of Operational Research 54(1): 81-94, 1991.

[35] Benjamin F. Hobbs and Jong-Shi Pang. Nash-cournot equilibria in electric power markets with piecewise linear demand functions and joint constraints. Operations Research 55(1): 113-127, 2007.

[36] Christian Kanzow and Daniel Steck. Augmented Lagrangian and exact penalty methods for generalized Nash equilibrium problems. SIAM Journal on Optimization 26(4): 2034-2058.

[37] Christian Kanzow and Daniel Steck. Augmented Lagrangian and exact penalty methods for quasi-variational inequalities. Computational Optimization and Applications 69(3): 801-824, 2018.

[38] Diethard Klatte and Wu Li. Asymptotic constraint qualifications and global error bounds for convex inequalities. Mathematical Programming 84(1): 137-160, 1999.

[39] Jacek B. Krawczyk. Numerical solutions to coupled-constraint (or generalised) Nash equilibrium problems. Computational Management Science 4(2): 183-204 2007.

[40] Jacek B. Krawczyk. Coupled constraint Nash equilibria in environmental games. Resource and Energy Economics 27(2): 157-181, 2005.

[41] Jacek B. Krawczyk and Stanislav Uryasev. Relaxation algorithms to find Nash equilibria with economic applications. Environmental Modeling and Assessment 5: 63-73 (2000).

[42] Ankur A. Kulkarni and Uday V. Shanbhag. On the variational equilibrium as a refinement of the generalized Nash equilibrium. Automatica 48(1) 45-55, 2012.

[43] Adrian S. Lewis and Jong-Shi Pang. Error bounds for convex inequality systems. In Generalized convexity, generalized monotonicity: recent results, pages 75-110. Springer, 1998.

[44] Koichi Nabetani, Paul Tseng, and Masao Fukushima. Parametrized variational inequality approaches to generalized Nash equilibrium problems with shared constraints. Computational Optimization and Applications 48(3):423-452, 2011. 
[45] Kung Fu Ng and Wei Hong Yang. Regularities and their relations to error bounds. Mathematical Programming 99(3):521-538, 2004.

[46] Hukukane Nikaido and Kazuo Isoda. Note on noncooperative convex games. Pacific Journal of Mathematics 5(Suppl. 1): 807-815, 1955.

[47] Jong-Shi Pang. Error bounds in mathematical programming. Mathematical Programming 79(1-3):299-332, 1997.

[48] Jong-Shi Pang and Masao Fukushima. Quasi-variational inequalities, generalized Nash equilibria, and multi-leader-follower games. Computational Management Science 2: 21-56, 2005. [Erratum: same journal 6: 373-375, 2009.]

[49] Jong-Shi Pang, Gesualdo Scutari, Francisco Facchinei, and Chaoxiong Wang. Distributed power allocation with rate constraints in gaussian parallel interference channels. IEEE Transactions on Information Theory 54(8):3471-3489, 2008.

[50] Jong-Shi Pang, Gesualdo Scutari, Daniel P. Palomar, and Francisco Facchinei. Design of cognitive radio systems under temperature-interference constraints: A variational inequality approach. IEEE Transactions on Signal Processing 58(6):3251-3271, 2010.

[51] Stephen M. Robinson. An implicit-function theorem for a class of nonsmooth functions. Mathematics of Operations Research 16(2): 292-309, 1991.

[52] J. Ben Rosen. Existence and uniqueness of equilibrium points for concave n-person games. Econometrica: Journal of the Econometric Society 33(3):520-534, 1965.

[53] Dand A. Schiro, Jong-Shi Pang, and Uday V. Shanbhag. On the solution of affine generalized Nash equilibrium problems with shared constraints by Lemke's method. Mathematical Programming, Series A 146(1):1-46, 2013.

[54] Oliver Stein and Nathan Sudermann-Merx. The noncooperative transportation problem and linear generalized Nash games. European Journal of Operational Research 266(2):543-553, 2018.

[55] Paul Tseng. Convergence of a block coordinate descent method for nondifferentiable minimization. Journal of Optimization Theory and Applications 109(3): 475-494, 2002.

[56] Anna von Heusinger and Christian Kanzow. Optimization reformulations of the generalized Nash equilibrium problem using nikaido-isoda-type functions. Computational Optimization and Applications 43(3):353-377, 2009.

[57] Jing-Yuan Wei and Yves Smeers. Spatial oligopolistic electricity models with Cournot generators and regulated transmission prices. Operations Research 47(1):102-112, 1999. 